\title{
AN ULTRASTRUCTURAL AND FUNCTIONAL ASSESSMENT OF NASAL CILIA AND SPERMATOZOA IN AN INFERTILE HUMAN MALE AND HEALTHY CONTROLS
}

\author{
by Lucy D. Harlow, William B. Watkins and Anthony Koutoulis
}

(with six plates and four tables)

\begin{abstract}
Harlow, L. D., Watkins, W. B. \& Koutoulis, A., 2004 (29.x): An ultrastructural and functional assessment of nasal cilia and spermatozoa in an infertile human male and healthy controls. Papers and Proceedings of the Royal Society of Tasmania 138: 1-10. https://doi.org/10.26749/rstpp.138.1 ISSN 0080-4703. Cell Biology Group, School of Plant Science, University of Tasmania, Private Bag 55, Hobart, Tasmania 7001, Australia (LDH, AK*); 141 Hampden Rd, Hobart, Tasmania 7000, Australia (WBW). * Author for correspondence.
\end{abstract}

\begin{abstract}
Ciliary disorders are complex and enigmatic, and can lead to infertility and respiratory complications. Human males who produce spermatozoa with defective flagella may also possess defective cilia. In this study, the motility and ultrastructure of nasal cilia/cells and spermatozoa of an infertile Tasmanian male patient were examined in order to elucidate the cause of his infertility. A detailed examination of the variation in cilia and spermatozoa of control subjects was also conducted. The axonemal structure of all nasal samples from control subjects showed a low frequency of abnormalities; however, when present, abnormalities varied between samples. The nasal cells and spermatozoa of the infertile patient were distinct from control samples, both in terms of motility and ultrastructure. The spermatozoa axonemes of the patient displayed the normal 9+2 microtubule ultrastructure; however, the proportion of immotile spermatozoa and ultrastructural abnormalities was higher than in control samples. All of the patient's nasal cilia were functionally immotile and approximately $70 \%$ of ciliated cells displayed extensive ciliary fusion; a feature not found in controls and not previously described. Despite having immotile nasal cilia, this patient was healthy with no obvious respiratory ailments. Additionally, the nasal cells of the patient possessed mitochondria with distended membranes, internalised membranous material, cilia with vesicles and internalised axonemes. These features suggest that ciliary deployment and membrane deposition was compromised in the patient.
\end{abstract}

Key Words: nasal cilia, spermatozoa, flagella, infertility.

\section{INTRODUCTION}

In humans, coordinated ciliary beating is important for clearing the airways of mucus and trapped foreign particles (Houtmeyers et al. I999, Ma et al. 1999), while the flagella of spermatozoa propel them to ova to facilitate fertilisation. Consequently, defects in cilia/flagella may be associated with respiratory ailments and lowered fertility, as well as impaired sensory organ and/or brain function (Afzelius et al. 1975, Afzelius \& Elaisson 1983, Mygind \& Pedersen 1983, Afzelius 1998). The ultrastructure of cilia and flagella are essentially identical and the terms are sometimes used interchangeably (Dentler \& Witman 1995), as is the case in this study.

Accurate diagnosis of ciliary disorders requires functional and ultrastructural analysis of cilia (Santamaria et al. 1999, Holzmann et al. 2000) from different systems (e.g., reproductive and respiratory) (Afzelius 1976, El-Sayed et al. 1997) and different areas within the one system as well as an understanding of the inherent variation that occurs within healthy populations (Nelson et al. 1995, Bush et al. 1998). Shared ultrastructural defects in cilia of different tissues would imply a genetic rather than an acquired disorder (Sturgess \& Turner 1990, de Longh \& Rutland 1995, Afzelius 1999).

This study examined the motility and ultrastructure of two different ciliated samples, nasal cells and spermatozoa. An infertile Tasmanian male patient who consistently produced an abnormally low proportion of motile spermatozoa was studied in order to elucidate the cause of his infertility, and examine whether his nasal cilia were affected. Samples from healthy subjects provided a standard from which to draw comparisons. The study aimed to quantify and describe variation in the axoneme and enhance our understanding of the role of cilia.

\section{MATERIALS AND METHODS}

\section{Nasal Cilia}

Ethics approval for this research was obtained from the Royal Hobart Hospital Ethics Committee, Hobart, Tasmania. Consent for biopsy of nasal cells was obtained from all participants. Nasal cells were collected from 13 control subjects (eight females and five males, aged 21-42) and a 37-year-old male infertile patient using the nasal brushing technique (Rutland et al. 1982). Neither the controls nor the patient had suffered recently from a respiratory illness, nor did they have a history of exposure to agents (particulate matter/chemicals and/or smoke) that may affect nasal cilia. Furthermore, the controls were non-smokers of whom none consumed more than an average of one to two glasses of alcohol per week. The patient consumed alcohol approximately once a fortnight and did not suffer from headaches, frequent colds or a persistent cough.

Individuals were requested to not consume: (i) caffeine for at least $4 \mathrm{~h}$ prior to sampling; or (ii) alcohol for at least $24 \mathrm{~h}$ prior to sampling (see Rossman et al. 1981). To increase tissue yield, patients blew their nose to clear excess secretions. A qualified physician performed the nasal brushings, using a $2 \mathrm{~mm}$ diameter nylon brush. The brush was first moistened in 5\% Delbecco's Minimum Essential Medium (DMEM) (GibcoBRL, USA) and the nasal passage brushed for a few seconds. Adherent epithelia were immediately dislodged from the brush by brisk agitation directly into a pre-warmed plastic vial containing $2 \mathrm{ml}$ of $5 \%$ DMEM. The brush was rinsed in $2 \mathrm{ml}$ of $2.5 \%$ glutaraldehyde in $0.1 \mathrm{M}$ Pipes buffer to fix the remaining cells for transmission electron microscopy (TEM). Post collection, cells in 5\% DMEM were kept in a $37^{\circ} \mathrm{C}$ water bath to permit inspection of live material by light microscopy. Cells in fixative were stored at $4^{\circ} \mathrm{C}$ until processed for TEM. 
Small strips of epithelia (up to $1 \mathrm{~mm}$ ) and/or individual nasal cells were observed under the light microscope immediately after collection. Ciliary beat frequency (CBF) (scored as [i] none, [ii] some or [iii] rapid), the proportion of ciliated cells to non-ciliated cells, cell morphology, ciliary morphology and beat pattern, and any abnormalities, were observed. The number of cilia per cell was recorded as normal or abnormal. Three slides of the same sample were examined such that an overall impression of the sample and the variability within the sample were obtained. Samples were recorded by video using a Nikon Labophot microscope. In all cases, pre-warmed $\left(37^{\circ} \mathrm{C}\right)$ slides and coverslips were used.

\section{Spermatozoa}

Semen samples were collected from two controls and the infertile patient. The control samples were from donors of an IVF programme and these have been used successfully in the programme. All specimens were collected after a minimum of $48 \mathrm{~h}$ but no longer than seven days of sexual abstinence, as recommended by the IVF laboratory. Samples collected away from the laboratory were delivered within $1 \mathrm{~h}$ of collection and specimens kept as close to body temperature as possible. Samples were collected into a sterile plastic container pre-warmed to body temperature. An aliquot of the semen was centrifuged at low speed for 5-10 min until a pellet had formed. The supernatant was removed and the pellet resuspended in $2.5 \%$ glutaraldehyde in $0.1 \mathrm{M}$ Pipes buffer and stored at $4^{\circ} \mathrm{C}$ until processed for TEM.

Macroscopic analysis of semen included appearance, volume and viscosity. The sample was allowed to liquefy at $37^{\circ} \mathrm{C}$ for a minimum of $15 \mathrm{~min}$ and mixed thoroughly with a swirling motion for $20-30 \mathrm{sec}$. The sample was examined immediately after liquefaction. A normal sample has a homogenous, gray opalescent appearance. The volume was measured with a sterile graduated pipette. Viscosity was estimated by allowing the semen to drop from the pipette and observing the drops or length of the thread. A sample with normal viscosity leaves the pipette with discrete drops. Increased viscosity can range from slightly increased to moderately increased, to grossly increased depending on the length of the thread (this can be up to $2 \mathrm{~cm}$ long). Viscosity may also be decreased if the semen is watery.

Light microscopy was used for detailed analysis. A $5 \mu \mathrm{l}$ drop of the sample was placed on a Makler chamber and the number of motile/non-motile spermatozoa was counted. A minimum of 200 spermatozoa were counted and the count repeated with a second aliquot of the sample (the count and motility were averaged). Progression or degree of forward movement was assessed according to the following scale: (i) poor - no forward motion (wavering) or slight forward motion; (ii) average - slow meandering forward motion; (iii) fair - more direct but slow forward motion; (iv) good - faster straight line motion; and (v) excellent - very rapid direct motion.

For detailed morphological observations, a slide was prepared from the raw semen sample. A drop of semen was placed between two slides. The slides were pulled apart gently and one smear was stained with hematoxylin and eosin, and fixed with DePeX mounting medium (Gurr, USA). Slides were left for a minimum of $24 \mathrm{~h}$ to set. Spermatozoa were documented as having a normal or abnormal morphology. Abnormalities were grouped into four categories: (i) cytoplasmic droplets; (ii) head defects; (iii) mid-piece defects; and (iv) tail defects. After initial observations spermatozoa were recorded by video using a Nikon Labophot microscope. In all cases, pre-warmed $\left(37^{\circ} \mathrm{C}\right)$ slides and coverslips were used. Slides of fixed spermatozoa were re-examined and more detailed observations of morphology conducted.

\section{Transmission Electron Microscopy (TEM)}

After initial fixation in $2.5 \%$ glutaraldehyde in $0.1 \mathrm{M}$ Pipes buffer, samples were washed three times for $3 \mathrm{~min}$ in $0.1 \mathrm{M}$ Pipes buffer, fixed in $1 \% \mathrm{OsO}_{4}$ in water for $2 \mathrm{~h}$, washed three times for $3 \mathrm{~min}$ in sterile water and stained in $4 \%$ aqueous uranyl acetate for $2 \mathrm{~h}$. Following staining, samples were washed three times for $3 \mathrm{~min}$ in sterile water and dehydrated in a graded series of ethanol $(25 \%, 50 \%$, $75 \%, 100 \%$ ) for 20 min per concentration. Two further 20 min-exposures to $100 \%$ ethanol were performed to ensure all water was removed. Cells were embedded in Spurr's resin (Spurr 1969) at 25\% increments $(25 \%, 50 \%, 75 \%$, $100 \%$ ) over a period of $2 \mathrm{~h}$. The $100 \%$ resin was changed twice, $20 \mathrm{~min}$ per change, followed by an overnight change and polymerised at $65^{\circ} \mathrm{C}$ for $25 \mathrm{~h}$. Ultrathin sections were stained for $30 \mathrm{~min}$ in $4 \%$ aqueous uranyl acetate, washed four times for $3 \mathrm{~min}$ in distilled water, stained in lead citrate for $3 \mathrm{~min}$, washed four times for $3 \mathrm{~min}$ in distilled water and examined in a Philips CM100 transmission electron microscope operated at $80 \mathrm{kV}$.

Initial observations consisted of documenting the variation in control ultrastructure, both of nasal cells and their associated cilia, and of spermatozoa and their associated cilium. For each sample, similarities and differences between control and the patient were documented. This included recording the percentage of apparently "normal" and "abnormal" cells and axonemes. All samples were examined by TEM. Extensive documentation was performed on seven randomly chosen control nasal samples and the two spermatozoa controls.

\section{RESULTS}

\section{Control Nasal Cilia}

The proportion of ciliated cells varied widely (15-80\%) between controls. All control samples possessed a very similar beat pattern, number of cilia per cell and CBF (scored as rapid). Control nasal cells viewed by electron microscopy possessed numerous regularly-spaced cilia, interspersed by branched microvilli (pl. 1A-D). Microvilli were present on regions of the cell surface both with and without cilia, and dominated at junctions between cells (pl. 1A). Cilia were 6 $\mu \mathrm{m}$ in length and $200 \mathrm{~nm}$ wide, while microvilli were considerably shorter, up to $2 \mu \mathrm{m}$ long, with a variable width. Microvilli sectioned towards their tip were 50-100 nm wide (pl. 1C), while microvilli sectioned at a branching point were up to $600 \mathrm{~nm}$ wide. The degree of branching varied from unbranched to six branches, with secondary branching common (pl. 1D).

The orientation of central pair (CP) microtubules of adjacent cilia was generally similar for controls; however, occasionally the CP microtubules were oriented in a different direction (pl. 1C). The ciliary membranes of control cilia were typically uniform in appearance (i.e., not "loose"or 


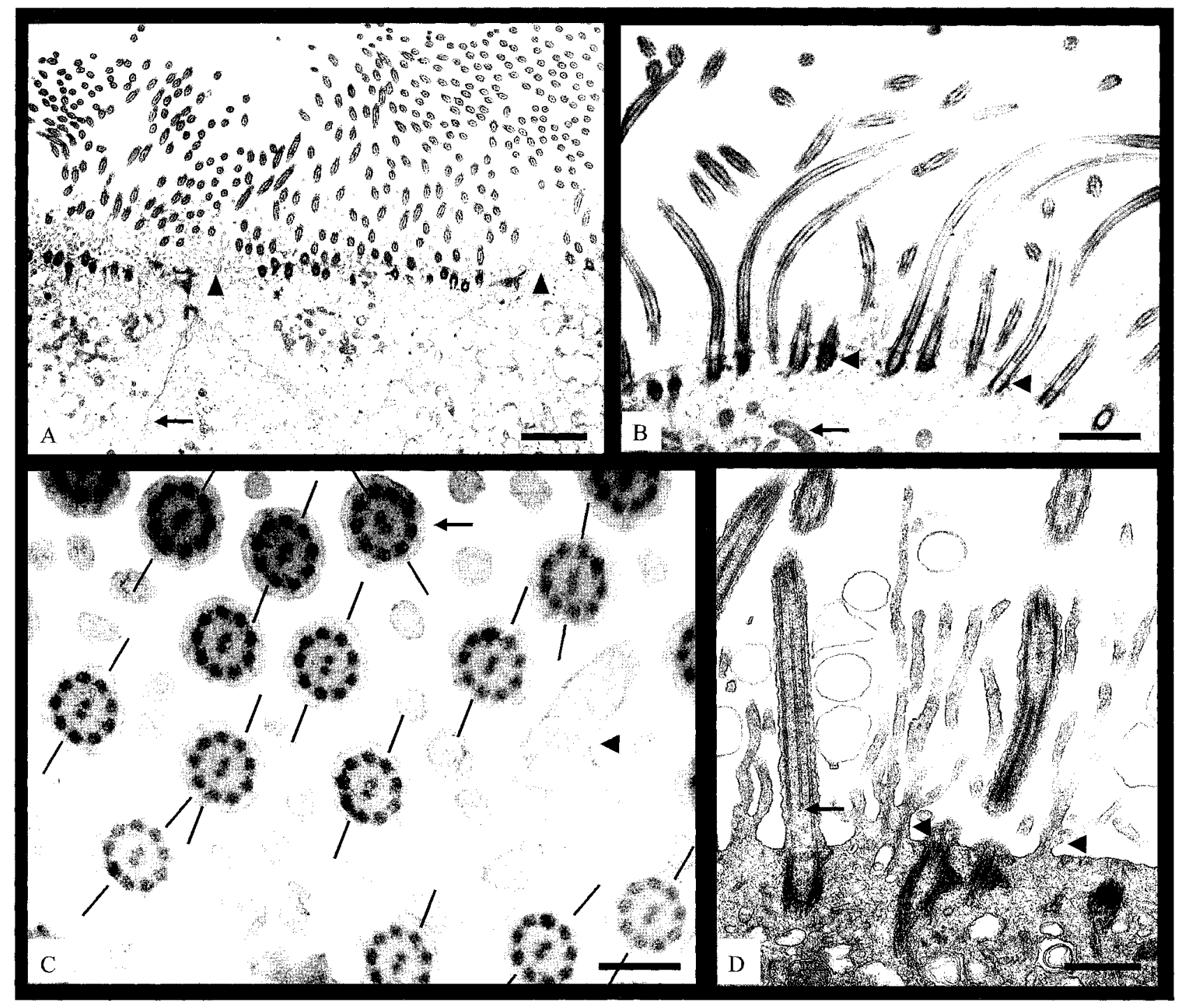

PLATE 1

Transmission electron micrographs of control nasal cells with cilia. (A) Oblique section showing numerous cilia and junctions between cells. Note cell membrane (arrow). Microvilli are interspersed between cilia at the cell surface and span the junction between cells (arrowheads). Scale bar $=2 \mu \mathrm{m}$. (B) Longitudinal section showing basal bodies (arrowheads). Note mitochondrion with uniform appearance of external and internal membranes (arrow). Scale bar $=1 \mu \mathrm{m}$. (C) Transverse section through a branched microvillus (arrowhead) and numerous cilia. The cilia possess the characteristic 9+2 microtubule arrangement. Ciliary orientation is defined by a line through the central pair microtubules. Note the cilium with misoriented central pair microtubules (arrow). Scale bar $=200 \mathrm{~nm}$. (D) Longitudinal section through branched microvilli (arrowheads) and cilia. The central pair microtubules of the ciliary axoneme begin some distance above the cell proper (arrow). Scale bar $=500 \mathrm{~nm}$.

wavy) (pl. 1C). Internal and external mitochondrial membranes in nasal cells also appeared uniform in controls (pl. IB).

The axonemal structure showed distinct changes along the length of the cilium. The CP microtubules of the axoneme were present $-350 \mathrm{~nm}$ from the cell proper (pl. 1D), forming the characteristic $9+2$ microtubular arrangement, consisting of nine outer doublet microtubules and two single CP microtubules. This arrangement continued up the length of the cilium until the tip, where the cilium tapered. In this region, microtubules were reduced in number and became disorganised resulting in a range of axonemal tip sections, all of which appeared abnormal (pl. 2A-D). Microtubule associated structures, such as dynein arms, occasionally persisted in tip regions (pl. 2C-D).
In all controls, microtubule associated structures (inner/ outer dynein arms, radial spokes, CP projections) were evident. Sections in which this detail could be seen were rare and could be documented only as present or absent. In the majority of sections, outer dynein arms were clearly visible. The proportion of normal axonemes $(9 \mathrm{~d}+2 \mathrm{cp}=9$ doublet microtubules with outer dynein arms +2 single CP microtubules), including tip sections, ranged from $81.8-94.9 \%$ in controls (table 1). A variety of different tip axonemal structures was observed in which single (s) microtubules were commonly present in place of doublet (d) microtubules and one or both CP microtubules were absent (pl. 2A). The following tip sections were observed in controls: $9 \mathrm{~d}+1 \mathrm{cp}, 9 \mathrm{~d}+0 \mathrm{cp}, 8 \mathrm{~d}+1 \mathrm{~s}+2 \mathrm{cp}, 8 \mathrm{~d}+2 \mathrm{cp}, 8 \mathrm{~d}+1 \mathrm{~s}+0 \mathrm{cp}$, $8 \mathrm{~d}+0 \mathrm{cp}, 7 \mathrm{~d}+2 \mathrm{~s}+0 \mathrm{cp}, 6 \mathrm{~d}+2 \mathrm{cp}, 5 \mathrm{~d}+4 \mathrm{~s}+2 \mathrm{cp}, 5 \mathrm{~d}+4 \mathrm{~s}+0 \mathrm{cp}$, $4 d+3 s+0 c p, 4 d+0 c p, 3 d+3 s+2 c p, 3 d+0 c p, 2 d+7 s+0 c p$, 


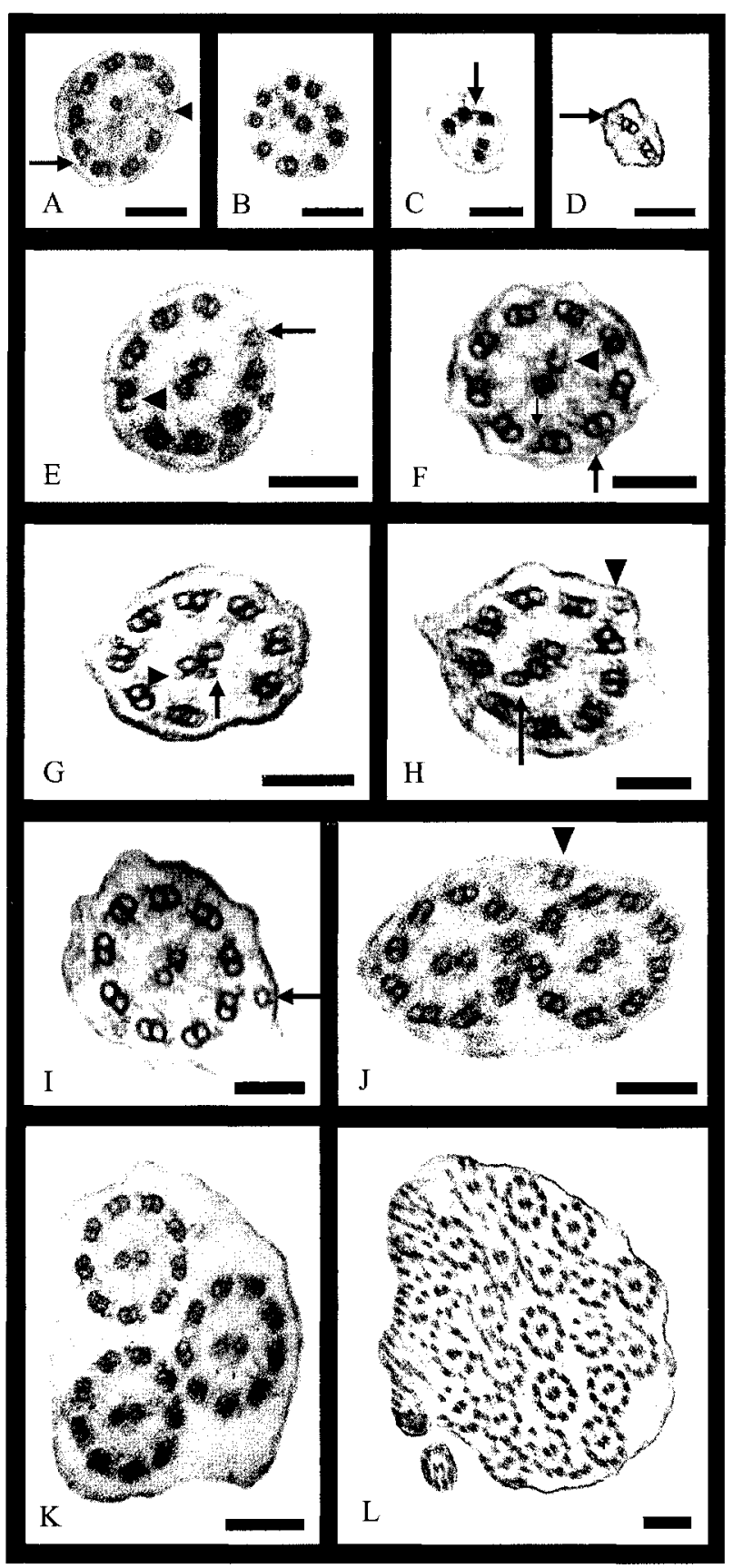

$2 \mathrm{~d}+3 \mathrm{~s}+2 \mathrm{cp}, 2 \mathrm{~d}+1 \mathrm{~s}+0 \mathrm{cp}, 2 \mathrm{~d}+0 \mathrm{cp}, 1 \mathrm{~d}+0 \mathrm{cp}, 9 \mathrm{~s}+2 \mathrm{cp}, 9 \mathrm{~s}+1 \mathrm{cp}$, $9 s+0 c p, 8 s+2 c p, 8 s+0 c p$ and $2-5$ random microtubules.

Ciliary abnormalities in controls occurred ar a low frequency $(10.4 \%$ ) (table 1 ). Microtubule abnormalities fell into three categories: (i) missing microtubules (1.8\%) (pl. 2E, G); (ii) misplaced microtubules $(0.2 \%)$; and (iii) extra microtubules, either inside (pl. 2H) or outside (pl. 2I) the axoneme $(0.8 \%)$. Incomplete or broken microtubules were observed; either the B-tubule of an outer doublet was not fully connected to the A-tubule (pl. 2E), or there was a break in a CP microtubule (pl. $2 \mathrm{~F}$ ). The most common abnormality observed in controls was compound cilia $(>1$ axoneme within the ciliary membrane). The extent of compound cilia ranged greatly in controls, from one and a half axonemes to over six (pl. 2J-L), with an average occurrence of $5.4 \%$ (table 1 ).

\section{PLATE 2}

Transmission electron micrographs of control nasal cilia exhibiting variation from the $9+2$ microtubule arrangement. (A) Axoneme with nine outer doublets, but only one of the two central pair microtubules. The nine outer doublets are not evenly spaced leading to a noticeable gap (arrowhead). Outer dynein arms can be seen (arrow). Scale bar $=100 \mathrm{~nm}$. (B) Axomene at the ciliary tip region with one outer doublet and eight outer singlets, all lacking dynein arms. The central pair microtubules are present, but off-centre. Scale bar $=100 \mathrm{~nm} .(\mathrm{C})$ Axoneme at the ciliary tip region with two singlet microtubules, possibly the central pair, and three outer doublet microtubules with outer dynein arms (arrow). Scale bar $=100 \mathrm{~nm}$. (D) Axoneme at the ciliary tip region with two outer doublets with outer dynein arms (arrow). Scale bar $=100 \mathrm{~nm}$. (E) Axoneme with eight outer doublets and one outer singlet (arrow). The B-tubule of one of the outer doublets is not completely associated with the A-tubule (arrowhead). Scale bar $=100 \mathrm{~nm}$. (F) Axoneme with nine outer doublets and two central pair microtubules. One of the central pair microtubules is incomplete (arrowhead). Some doublets lack outer dynein arms (arrows). Scale bar $=100 \mathrm{~nm}$. (G) Axoneme with eight outer doublets, abnormal placement of central pair projection (arrowhead) and an additional small microtubule-like structure (arrow) associated with the central pair. Scale bar $=100 \mathrm{~nm}$. (H) Axoneme with nine outer doublets, two central pair microtubules and two additional single microtubules; one near the central pair microtubules (arrow) and one near the ciliary membrane (arrowhead). Scale bar $=100 \mathrm{~nm}$. (I) Axoneme with nine outer doublets, two central pair microtubules and an additional single microtubule (arrow). Scale bar $=100 \mathrm{~nm}$. (J) Compound cilium with two axonemes, each consisting of a $9+2$ microtubule arrangement, and an additional single microtubule (arrowhead). Scale bar $=100 \mathrm{~nm}$. $(K)$ Compound cilium with three axonemes, each consisting of a 9+2 microtubule arrangement. Scale bar $=$ $100 \mathrm{~nm}$. (L) Compound cilium with numerous axonemes. Scale bar $=200 \mathrm{~nm}$.

\section{Patient Nasal Cilia}

The nasal cells and nasal cilia of the patient showed an unusual morphology by light microscopy. Approximately 30\% of ciliated cells displayed varying degrees of fusion of cilia, ranging from what appeared to be separate cilia with normal morphology as seen in controls to partially fused cilia (pl. 3A, B), while the majority of ciliated cells $(-70 \%)$ possessed cilia that appeared completely fused (pl. 3C). Fused cilia did not beat, while the tips of some non-fused cilia showed tiny abnormal movements ( $\mathrm{CBF}$ scored as none), only detectable upon detailed examination of video recordings. Therefore, all nasal cilia in the patient were deemed functionally immotile. Fused cilia were not found in any controls.

The patient possessed cilia with the $9+2$ axonemal structure and the appropriate microtubule associated structures. However, the patient possessed an unusually low number of normal axonemes, only $39 \%$ compared to the 
TABLE 1

Comparison of control and patient nasal ciliary ultrastructure

\begin{tabular}{lcc}
\hline & Control (range) & Patient \\
\hline \% Normal TS* (no tips) & 69.7 & 19.5 \\
\% Normal TS & $(46.2-89.3)$ & \\
& 89.6 & 39 \\
\% Abnormal TS & $10.4(5.1-18.2)$ & 61 \\
\% Half axoneme & 0 & 4.8 \\
\% Internalised microtubules & 0 & 9.7 \\
\% Missing microtubules & $1.8(0-10.8)$ & 8.1 \\
\% Misplaced microtubules & $0.2(0-1.1)$ & 3.7 \\
\% Extra-numerary microtu- & $0.8(0-2.5)$ & 1.6 \\
bules & & \\
\% Ciliary vesicles & $1.8(0-6.1)$ & 45.2 \\
\% Compound (>1 axoneme) & $5.4(2.3-11.5)$ & 7.8 \\
\% Abnormal TS excluding & $5(0-15.9)$ & 53.2 \\
compound & & \\
Total \# TS examined & $405(58)$ & 77 \\
\hline
\end{tabular}

${ }^{*} \mathrm{TS}=$ transverse sections

control mean of $89.6 \%$ (table 1). Furthermore, abnormal cilia were six times more frequent than the control mean (table 1). Although unfused cilia were found ( $\mathrm{pl} .4 \mathrm{~A}$ ), the high number of abnormal cilia in the patient was largely due to ciliary fusion ( $\mathrm{pl} .4 \mathrm{~B}, \mathrm{C}$ ) and the presence of vesicles between the axoneme and ciliary membrane ( $\mathrm{pl}$. 4D). The patient displayed microtubule abnormalities not found in controls such as half axonemes and off-centre CP microtubules. Additionally, the patient possessed cells with a high proportion of internal microtubules and/or internal axonemes (pl. 4E, table 1).

Ciliary abnormalities found in the patient, at a frequency greater than the control range, included misplaced microtubules and ciliary vesicles. Cilia of the patient also possessed "loose" ciliary membranes giving a wavy appearance (pl. 4F), while cells possessed distended mitochondria (pl. $4 \mathrm{~A}$ ) and significant amounts of internalised membraneous or fibrous material (pl. $4 \mathrm{G}$ ). The frequency of compound cilia in the patient $(7.8 \%)$ was within the control range (2.3-11.5\%) (table 1). Mis-orientation of adjacent cilia (pl. $4 \mathrm{~F}$ ) occurred at a slightly higher frequency in the patient than controls.

\section{Control and Patient Spermatozoa}

The patient possessed an unusually low proportion $(33 \%)$ of motile spermatozoa compared to the control mean (69\%) and possessed spermatozoa with a low rate of forward movement or "progression" (table 2). Furthermore, his spermatozoa count was unusually low ( - half the control mean) and his semen volume was unusually low $(0.5 \mathrm{ml}$ compared to a control average of $5.4 \mathrm{ml}$ ) (table 2).

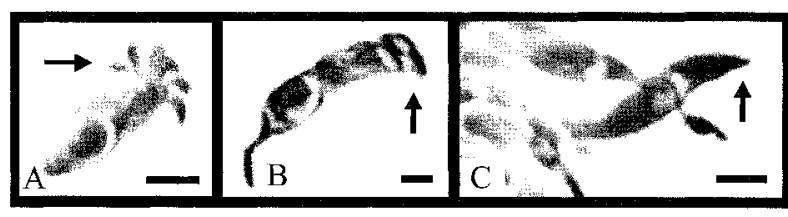

PLATE 3

Light microscope images of ciliated nasal cells of the patient showing different degrees of ciliary fusion. All scale bars = $4 \mathrm{um}$. (A) Cilia (arrow) with apparent normal morphology (i.e., separate cilia), as seen in control samples. (B-C) Nasal cells with different degrees of ciliary fusion (arrows).

At the light microscope level, some spermatozoa in the patient's samples displayed the same appearance as normal spermatozoa in control samples (pl. 5A). Spermatozoa often displayed multiple defects. The proportion of patient spermatozoa with an abnormal morphology (78\%) was within the control range $(78-83 \%)$ (table 3$)$. A range of different head abnormalities was observed (pl. 5B, C). Unlike controls, some patient spermatozoa possessed cytoplasmic droplets, which accounted for $1 \%$ of abnormal spermatozoa (table 3). Cytoplasmic droplets were either distending from the acrosome or the mid-piece region. Spermatozoa with pin heads (pl. 5B), double heads, double tails, short tails (pl. 5D, G) and mid-piece abnormalities (pl. 5B, D-G) were found in control and patient samples.

Spermatozoa examined by TEM (pl. 6) were analysed according to whether they were sectioned transversely (pl. 6A) or longitudinally (pl. 6B) (table 4). Spermatozoa with internal axonemes formed a large proportion of the abnormalities in the patient and the controls; these internal axonemes were often accompanied by the outer thick fibre, and mid-piece mitochondria or tail region fibrous sheaths typical of human sperm. For instance, $73.1 \%$ of the patient's spermatozoa possessed internal axonemes ( $\mathrm{pl}$. $6 \mathrm{~F}, \mathrm{G}$ ), while $57.9 \%$ of control spermatozoa possessed internal axonemes (pl. 6C) (table 4). The percentage of the patient's spermatozoa with a normal ultrastructure in longitudinal view (17.9\%) was considerably lower than the control range (56.7-60.5\%) (table 4). This was also the case in transverse view; $29 \%$ in the patient and $34-50 \%$ in controls (table 4).

Microtubule abnormalities could be evaluated more easily in transverse section than longitudinal section and were grouped into a range of sub-categories (table 4), which could be scored more than once if an axoneme possessed multiple defects or an axoneme was sectioned more than once (e.g., internalised axonemes). Generally, the patient possessed a higher percentage of defects compared to the controls. Compound cilia were rare in controls $(0.75 \%)$, but more common in the patient (6.8\%) (table 4). Unlike nasal cilia, the only form of compound cilia found in spermatozoa was those with two axonemes within the ciliary membrane. Other infrequent abnormalities found in controls included twisting of the outer doublet microtubules and unusually wide cilia (pl. 6D).

The patient also possessed unusually wide cilia; however, unlike controls, microtubules were sometimes absent from these cilia (pl. 6G). The fibre bundles of the cilium displayed a similar set of abnormalities as the axoneme. Fibres were documented as missing, misplaced, extra-numerary ( $\mathrm{pl}$. $6 \mathrm{E})$ or internalised. 


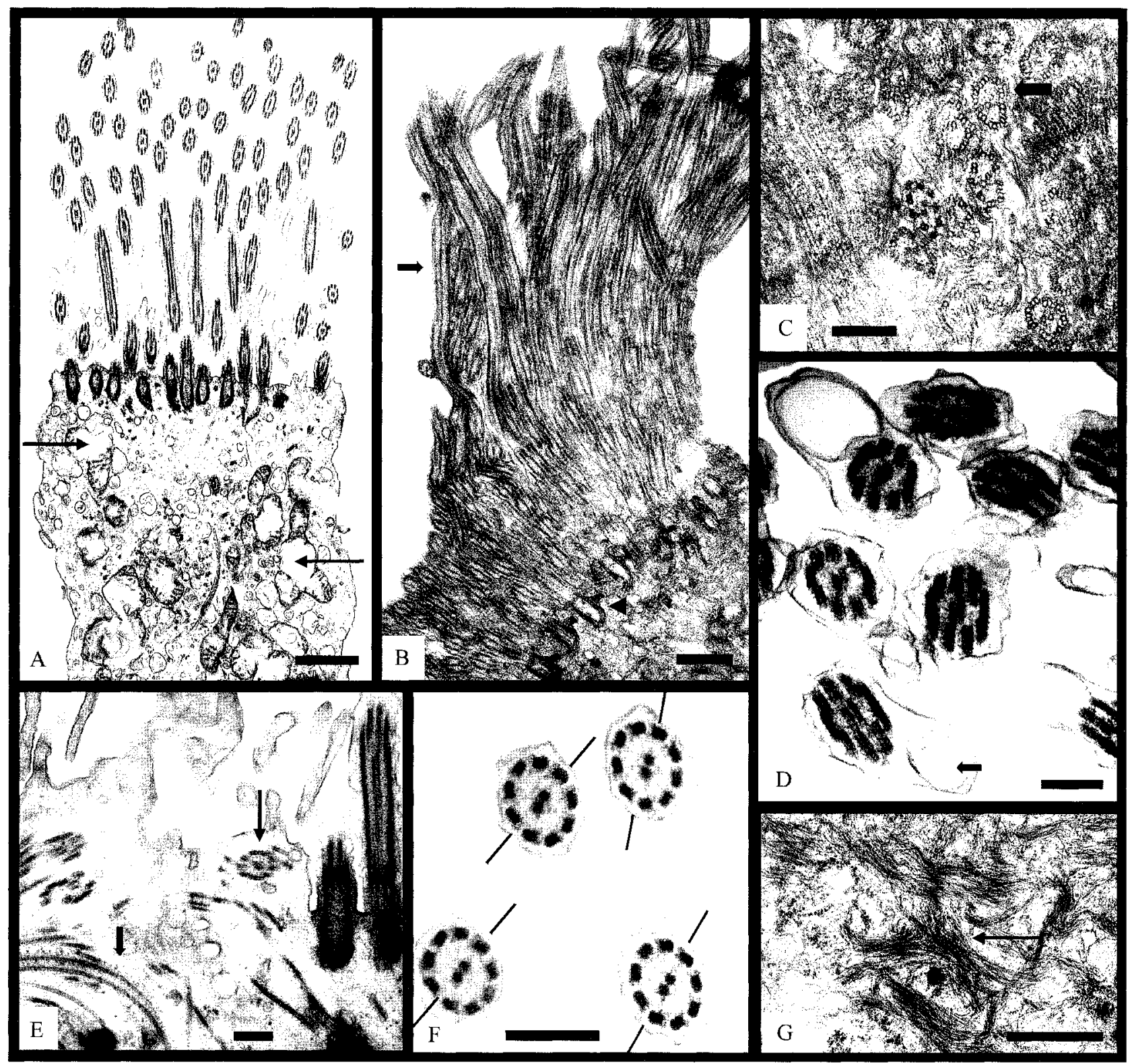

PLATE 4

Transmission electron micrographs of patient nasal cells and cilia. (A) Lonoitudinal section of nasal cell with apparently normal ciliary arrangement. Cilia are not fused and there are no vesicles within the ciliary membrane; however, mitochondria possess distended membranes (arrows). Scale bar $=1 \mathrm{\mu m}$. (B) Nasal cell with fused cilia (arrow). Note basal bodies (arrowhead). Scale bar $=500 \mathrm{~nm}$. (C) Glancing section of fused cilia. Some axonemes are sectioned transversely and exhibit the 9+2 microtubule arrangement (arrow). Axonemes are intertwined with additional membranous material. Scale bar $=200 \mathrm{~nm}$. (D) Cilia with vesicles within ciliary membrane (arrow). Scale bar $=200 \mathrm{~nm}$. (E) Nasal cell with numerous internalised microtubules (short arrow) and internalised axonemes with $9+2$ microtubule arrangement (long arrow). Scale bar $=200 \mathrm{~nm}$. (F) Transverse section through cilia with normal microtubular arrangment, normal ciliary orientation (as determined by the lines) and the presence of dynein arms, radial spokes and central pair projections. Scale bar $=200 \mathrm{~nm}$. $(G)$ Copious membranous or frbrous material (arrow) within a nasal cell. Scale bar $=500 \mathrm{~nm}$. 


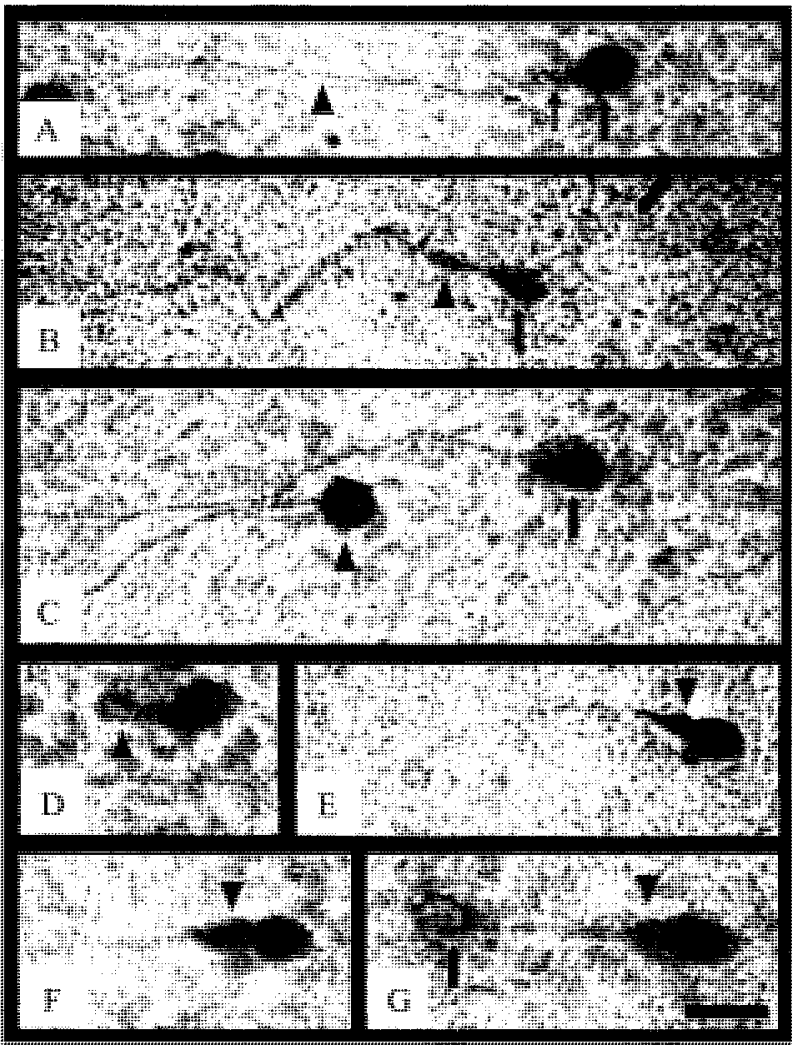

PLATE 5

Light microscope images of fixed and stained spermatozo a from the patient. Scale bar for all images $=5 \mu \mathrm{m}$. (A) Normal spermatozoon as seen in control samples. Note the three distinct regions of the cell: acrosome (thick arrow), mid-piece (thin arrow) and tail (arrowhead). (B)-( $G$ ) Defective spermatozoa which can also be found in control samples. (B) Spermatozoon with pin-head (arrow) and thick mid-piece (arrowhead). (C) Spermatozoa with abnormally shaped heads: one head is unusually tapered (arrow), while the other is more spherical (arrowhead). (D) Spermatozoon with a stumpy tail (arrowhead) that may be internalised. (E) Spermatozoon with enlarged midpiece (arrowhead). (F) Spermatozoon with enlarged mid-piece (arrowhead). Note the enlargement is far more pronounced than the spermatozoon shown in $(E)$. (G) Spermatozoon with enlarged mid-piece (arrowhead) and coiled tail (arrow).

TABLE 2

Macroscopic examination and light microscopy of semen

\begin{tabular}{lll}
\hline & \multicolumn{1}{c}{ Control (range) } & Patient (range) \\
\hline \# Samples averaged & 2 & 7 \\
\% Motility & $69(64-74)$ & $33(14-49)$ \\
Progression $(1-5)$ & Good $(4-4)$ & Fair $(3-3)$ \\
Count $\left(\times 10^{6} \mathrm{ml}^{-1}\right)$ & $93(52-168)$ & $48(31-93)$ \\
Volume $(\mathrm{ml})$ & $5.4(3.6-7)$ & 0.5 \\
Viscosity & Normal-moderately & Slightly increased* \\
& increased & \\
\hline
\end{tabular}

*Within control range
TABLE 3

Light microscope evaluation of the morphology of control and patient spermatozoa

\begin{tabular}{|c|c|c|}
\hline & Controls (tange) & Patient (range) \\
\hline \# Samples averaged & 2 & 3 \\
\hline$\%$ Normal forms & $19.5(17-22)$ & $22(21-24)$ \\
\hline$\%$ Abnormal forms & $80.5(78-83)$ & 78 \\
\hline$\%$ Cytoplasmic droplet & 0 & $1(0-3)$ \\
\hline$\%$ Abnormal head & $80(78-82)$ & $64.7(59-70)$ \\
\hline$\%$ Abnormal mid-piece & $0.5(0-1)$ & $12.3(9-14)$ \\
\hline$\%$ Abnormal tai ${ }^{*}$ & 0 & 0 \\
\hline
\end{tabular}

*Some spermatozoa with abnormal tails also fell into the abnormal head and/or abnormal mid-piece categories, and these were not scored twice.

TABLE 4

Comparison of control and patient spermatozoa ultrastructure

\begin{tabular}{|c|c|c|}
\hline & Control (range) & Patient \\
\hline $\begin{array}{l}\text { \# Cells/axonemal longi- } \\
\text { tudinal sections }\end{array}$ & $34(30-38)$ & 67 \\
\hline $\begin{array}{l}\% \text { Cells with internalised } \\
\text { axonemes }\end{array}$ & $57.9(50-65.8)$ & 73.1 \\
\hline$\%$ Normal axonemes & $58.6(56.7-60.5)$ & 17.9 \\
\hline$\%$ Abnormal axonemes & $41.4(39.5-43.3)$ & 82.1 \\
\hline $\begin{array}{l}\% \text { Cells with internalised } \\
\text { microtubules }\end{array}$ & $10.4(5.3-13.3)$ & 17.9 \\
\hline $\begin{array}{l}\text { \# Axonemal transverse } \\
\text { sections excluding tips }\end{array}$ & $59.5(53-66)$ & 74 \\
\hline$\%$ Normal axomenes & $42(34-50)$ & 29 \\
\hline$\%$ Compound ( $>1$ axoneme) & $0.75(0-1.5)$ & 6.8 \\
\hline$\%$ Half axoneme & $11.7(4.5-18.9)$ & 25.7 \\
\hline$\%$ Missing microtubules & $26.5(22.7-30.2)$ & 29.7 \\
\hline $\begin{array}{l}\% \text { Extra-numerary } \\
\text { microtubules }\end{array}$ & $0.95(0-1.9)$ & 4.1 \\
\hline $\begin{array}{l}\% \text { Disorganised micro- } \\
\text { tubules }\end{array}$ & $13.8(10.6-17)$ & 35.2 \\
\hline$\%$ Abnormal fibres** & $2.25(0-4.5)$ & 8.1 \\
\hline$\%$ Other ${ }^{* * *}$ & $0.95(0-1.9)$ & 3.3 \\
\hline
\end{tabular}

Note: percentage of axonemal abnormalities in transverse section exceeds 100 because some axonemes possessed more than one defect.

* Includes axonemes with misplaced microtubules, offcentre CP and CP too close.

** Includes missing, misplaced, extra-numerary and internalised fibres.

*** Includes abnormally wide cilia, ciliary vesicles, twisted outer doublets (LS) and B-tubule broken off Atubule. 


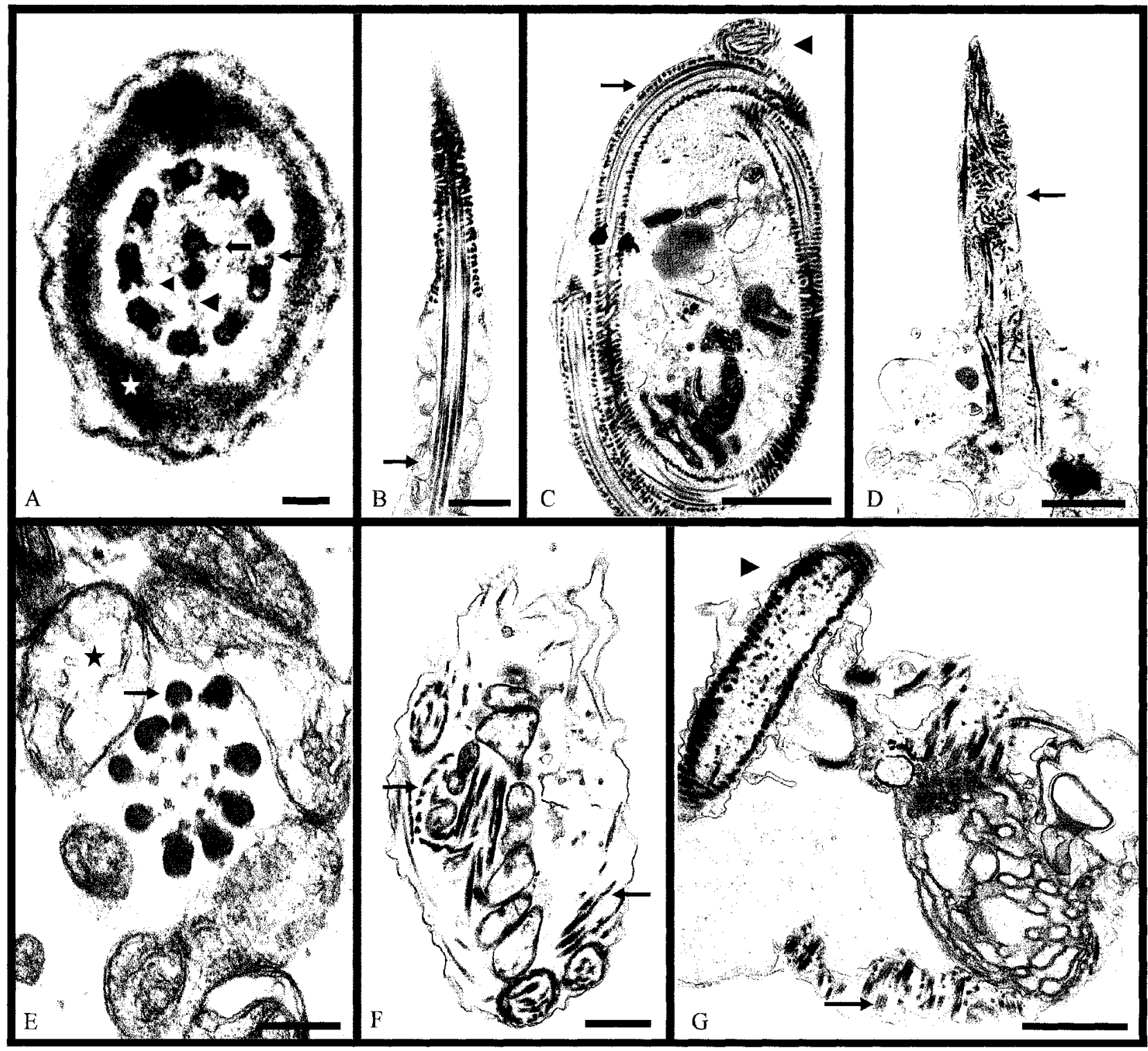

PLATE 6

Transmission electron micrographs of spermatozoa. (A) Transverse section through a control cilium with a $9+2$ microtubule arrangement surrounded by a fibrous sheath (star). Note microtubule projections, including outer dynein arms (thin arrow), inner dynein arms (small arrowhead) radial spokes (large arrowhead) and central pair projections (thick arrow). Scale bar $=$ $50 \mathrm{~nm}$. (B) Longitudinal section through a control cilium at the junction between the mid-piece (with mitochondrial profiles [arrow]) and the tail region (with fibrous sheath [top of section]). Scale bar $=500 \mathrm{~nm}$. (C) Control spermatozoon showing variation. Note coiled internalised axoneme sectioned longitudinally (arrow) and almost transversely (arrowhead). Scale bar = $1 \mathrm{um}$. (D) Control spermatozoon showing variation. Note short broad cilium containing extra-numerary, apparently disorganised microtubules or fibrous sheath components (arrow). Scale bar $=1 \mu \mathrm{m}$. (E) Transverse section through basal region of a cilium from a patient spermatozoon showing an additional or split fibre bundle (arrow). Mitochondria surround the axoneme (star). Scale bar $=250 \mathrm{~nm}$. (F) Section through a patient spermatozoon showing internalised microtubules (arrows), some of which may represent axonemal profiles. Scale bar $=500 \mathrm{~nm}$. (G) Section through a patient spermatozoon showing internalised axoneme that is twice the width of a normal cilium and lacks microtubules (arrowhead). Scale bar $=500 \mathrm{~nm}$.

\section{DISCUSSION}

\section{Patient Summary}

The nasal cells/cilia and spermatozoa of the patient were functionally immotile but this was not attributable to a lack of co-ordination (misorientation of ciliary axonemes) or an axonemal defect(s). The patient's cilia were clearly distinct from controls and displayed a higher frequency of abnormalities at the ultrastructural level. Strikingly, the patient's nasal cells displayed extensive ciliary fusion, vesicles within the ciliary membrane, distended mitochondria and internal microtubules/axonemes (also in spermatozoa). These observations suggest that membrane deposition or deployment is compromised in the patient resulting in $a b$ normal ciliogenesis. Internalised axonemes may result from a lack of ciliary deployment; vesicles may be a product of excess ciliary membrane; mitochondrial membranes may be 
produced copiously or malformed, resulting in the observed distention; and excess membranes within the cell proper may cause ciliary axonemes to become matted or "fused". Unlike other membrane defects such as cilia with finlike extensions (Herzon 1983), individual membranes were absent from fused axonemes such that these cilia were unable to beat. Therefore, although the patient does not have an axonemal problem per se, his ciliated cells were affected.

The cause of the patient's infertility was less obvious than the immotility of his nasal cilia. Male infertility is complex and men can often have spermatozoa with an impaired motility but an apparently "normal" ultrastructure (Greenstone et al. 1983, Santamaria et al. 1999), or vice versa (de Longh \& Rutland 1995). It is known that the spermatozoa of the patient are incapable of fertilising an ovum under normal conditions, while those of the two donor controls are capable of fertilisation. Distinct features of the patient's semen were the: (i) extremely low volume collected; (ii) high proportion of immotile spermatozoa and spermatozoa with reduced progression; (iii) unusually low number of spermatozoa; and (iv) unusually high proportion of spermatozoa with an abnormal ultrastructure. It appears that the high proportion of immotile spermatozoa in the patient's samples reflects the high proportion of spermatozoa with internalised axonemes and the degree of internalisation. It is presumed that spermatozoa with internal axonemes would be functionally immotile and hence incapable of fertilisation. More specific axonemal abnormalities did not appear to be connected to patient infertility.

\section{Control Ciliary Variation}

It is expected that ciliary abnormalities found in control subjects are an attribute of environmental factors, and are therefore acquired. The main causes of acquired ciliary defects are infectious or toxic exposures (Sleigh 1983, Toremalm 1983). Damage commonly arises as a result of cigarette smoke (Fox et al. 1983, Knoll \& Talbot 1998), alcohol and/or caffeine (Johnsen et al. 1974). In addition, some local anaesthetics (Afzelius \& Elaisson 1983) as well as bacterial (de Longh \& Rutland 1995, Nelson et al. 1995) and fungal (Pieckova \& Jesenska 1998) products temporarily inhibit ciliary motility. Infections may cause ciliary damage but do not appear to affect spermatozoa motility (Afzelius 1998). Such factors may account for the baseline level of ciliary disorientation, compound cilia (Nielsen et al. 1983), and the wide variability in the types of ciliary abnormalities found in control subjects and the patient.

Caution is required when determining diagnostic features. Previous studies have not looked extensively at control variation in nasal samples. Certain microtubule arrangements documented in controls in this study (e.g., axonemal sections with only two doublet microtubules) have been reported as abnormal by previous studies (Arden \& Fox 1979) when these arrangements are the natural tapering of the cilium at its tip. Interestingly, branching microvilli, a "normal" feature of nasal cells, do not appear to have been previously documented.

The average frequency of abnormal nasal cilia for controls was $10.4 \%$ compared to $1 \%$ (Levison et al. 1983 ) and < $5 \%$ (Rossman et al. 1983) for previous studies. This may reflect the range of variation in controls that has in the past been attributed to ciliary disorders. Similarly, control spermatozoa exhibited a range of abnormalities both externally (i.e., at the light microscope level) and internally (i.e., at the TEM level). This seemingly high proportion of abnormal spermatozoa reflects the high IVF laboratory standards for spermatozoa with a normal morphology. The main abnormality of control spermatozoa, observed at the TEM level, was internalised axonemes (i.e., a tail defect). When these spermatozoa are observed under the light microscope they appear to have an abnormally large head and acrosome region (i.e., a head defect). Current clinical diagnosis of infertility may therefore be biased towards head defects.

\section{Defining Fused Cilia}

Large cilia have been described previously in the form of compound cilia (a term used interchangeably with fused cilia and megacilia) of various forms (Takasaka et al. 1980, Corbeel et al. 1981, Wright 1982, Bryan 1983 [not illustrated], Zahm et al. 1990 [no TEM images]), cilia with cyst-like structures (Tsang et al. 2000) and so-called fusion cilia (Herzon 1983). These fusion cilia are not the same as those described in our study. Fusion cilia, whether complete or partial, are usually not much larger than a single $9+2$ size (Herzon 1983). Although membrane defects such as finlike extensions from the ciliary membrane or defects of the shaft membrane have been described (Herzon 1983), the fused cilia in our study do not resemble these, nor are they compound cilia. Use of the term "fused" in literature is inconsistent and needs clarification. The fused cilia in our study do not appear to have been previously documented and may represent a novel disorder. Extensive research would be necessary to determine how common this disorder may be.

\section{Conclusions}

Interpretation of these results is complex and warrants further study, given the inability to analyse data statistically because of a single experimental subject. In particular, study of control variation and the effect of fused cilia on mucociliary clearance would be rewarding. Continuation of this work could involve examining a broader range of samples, with a particular focus on membranes. It is important to note that spermatozoa possess only one cilium (flagellum) such that ciliary fusion cannot occur.

It would have been interesting to include relatives of the patient in further genetic studies. However, during the course of this research, the male patient's partner was successful in becoming pregnant using IVF technology. Once this occurred, the male patient did not want to participate in any further research. Potentially, the infertility of this patient represents a novel disorder. Interestingly, the patient leads a healthy life and does not suffer from symptoms of impaired ciliary function (such as respiratory infections, coughs, frequent colds or headaches). He describes himself as being quite fit, and it could be that his fitness compensates for the defects found in this study. Similarly, organs that possess axonemal structures such as sensory organs (e.g., auditory hair cells and photoreceptors of the eye) do not appear affected (i.e., vision, balance and hearing were not impaired). The patient's anatomy displayed the normal left-right asymmetry.

Clearly there is much to learn about: (i) the functioning of respiratory cilia and their significance for healthy airways; and (ii) the complex factors that affect fertility. Interestingly, 
examination of nasal cilia may facilitate clinical diagnosis of infertility, specifically in males whose spermatozoa have lowered motility and abnormal function.

\section{ACKNOWLEDGEMENTS}

We thank the patient and volunteers who acted as controls in this study. We also thank Dr Tara Andersen at the Royal Hobart Hospital, Tasmania, for performing the nasal brushings and IVF laboratory staff at the St Helen's Private Hospital, Tasmania, for their assistance.

\section{REFERENCES}

AfzElius, B.A., 1976: A human syndrome caused by immotile cilia. Science 193: 317-319.

AFZFLIUS, B.A., 1998: Immotile cilia syndrome: past, present, and prospects for the future. Thorax 53: 894-897.

AFzelius, B.A., 1999: Asymmetry of cilia and of mice and men. International Journal of Developmental Biology 43: 283-286.

AfZELIUS, B.A. \& EI AISSON, R., 1983: Male and female infertility problems in the immotile-cilia syndrome. European Journal of Respiratory Disease 64: 144-147.

AfZei.jus, B.A., Ellasson, R., JOHnsen, O. \& Lindholmer, C., 1975: Lack of dynein arms in immotile human spermatozoa. Journal of Cell Biology 66: 225-232.

ARDEN, G.B. \& FOX, B., 1979: Increased incidence of abnormal nasal cilia in patients with retinitis pigmentosa. Nature 279: 534-536.

BRYAN, J.H.D., 1983: Abnormal cilia in a male-sterile mutant mouse. Virchows Archiv 400: 77-86.

Bush, A., Cole, P., Hariri, M., Mackay, I., Phillips, G., O'CAllaghan, C., Wilson, R. \& Warner, J.O., 1998: Primary ciliary dyskinesia: diagnosis and standards of care. European Respiratory Journal 12: 982-988.

Corbeel, l., Cornillie, F., Lauweryns, J., Boel, M. \& Van DEN BERGHE, G., 1981: Ultrastructural abnormalities of bronchial cilia in children with recurrent airway infections and bronchiecrasis. Archives of Disease in Childhood 56: 929-933.

DE LonGH, R. \& RUTI.AND, J., 1995: Ciliary defects in healthy subjects, bronchiectasis, and primary ciliary dyskinesia. American Joumal of Respiratory and Critical Care Medicine 151: $1559-1567$.

Dentler, W. \& Witman, G., 1995: Cilia and flagella. Methods in Cell Biology 47. Academic Press, San Diego.

El-SaYed, Y., Al-SARhani, A. \& Al-Essa, A.R., 1997: Otological manifestations of primary ciliary dyskinesia. Clinical Otolaryngology and Allied Sciences 22: 266-270.

FoX, B., BULL, T.B. \& Oliver, T.N., 1983: The distribution and assessment of electron-microscopic abnormalities of human cilia. European Journal of Respiratory Disease 64: 11-18.

Greenstone, M.A., Dewar, A. \& Cole, P.J., 1983: Ciliary dyskinesia with normal ultrastructure. Thorax 38: 875-876.

HFrzon, F.S., 1983: Nasal ciliary structural pathology. Laryngoscope 93: 63-67.

Holzmann, D., OTT, P.M. \& Felix, H., 2000: Diagnostic approach to primary ciliary dyskinesia: a review. European Journal of Pediatrics 159: 95-98.

Houtmeyers, E., Gosselink, R., Gayan-Ramirez, G. \& DeCRAMER, M., 1999: Effects of drugs on mucous clearance. European Respiratory Journal 14: 452- 467.

Johnsen, O., Eldasson, R. \& Abdel Kader, M.M., 1974: Effects of caffeine on the motility and metabolism of human spermatozoa. Andrologia 6: 53.

KNOLL, M. \& TALBOT, P., 1998: Cigarette smoke inhibits oocyte cumulus complex pick-up by the oviduct in vitro inde- pendent of ciliary beat frequency. Reproductive Toxicology 12: $57-68$.

Levison, H., Mindorff, C.M., ChaO, J., Turner, J.A.P., STURGESS, J.M. \& SIRINGER, D.A., 1983: Pathophysiology of the ciliary motility syndromes. European Journal of Respiratory Disease 64: 78-90.

Ma, W., Korngreen, A., UZlaner, N., Priel, Z. \& SilberBERG, S.D., 1999: Extracellular sodium regulates airway ciliary motility by inhibiting a P2X receptor. Nature 400 : 894-897.

Mygind, N. \& Pedersen, M., 1983: Nose-, sinus- and earsymptoms in 27 patients with primary ciliary dyskinesia. European Journal of Respiratory Disease 64: 96-101.

Nelson, S., Mason, C.M., KOLLS, J. \& SuMmer, W.R., 1995; Pathophysiology of preumonia. Clinics in Chest Medicine 16: $1-12$.

Nielsen, M.H., Pedersen, M., Christensen, B. \& Mygind, N., 1983: Blind quantitative electron microscopy of cilia from patients with primary ciliary dyskinesia and from normal subjects. European Journal of Respiratory Disease 64: 19-30.

Pifckova, E. \& Jesenska, Z., 1998: Molds on house walls and the effect of their chloroform-extractable metabolites on the respiratory cilia movement of one-day-old chicks in vitro. Folia Microbiologica 43: 672-678.

Rossman, C.M., FOrREsT, J.B., LEe, R.M.K.W., NEWhOUSE, A.F. \& Newhouse, M.T., 1981: The dyskinetic cilia syndrome: abnormal ciliary motility in association with abnormal ciliary ultrastructure. Chest 80: 860-863.

Rossman, C.M., LeE, R.M.K.W., ForRest, J.B. \& Newhouse, M.T., 1983: Nasal cilia in normal man, primary ciliary dyskinesia: analysis of motility and ultrastructure. European Journal of Respiratory Disease 64: 64-70.

Rutland, J., Dewar, A., Cox, T. \& Col.f. J., 1982: Nasal brushing for the study of ciliary ultrastructure. Journal of Clinical Pathology 35: 357-359.

Santamaria, F., De Santi, M.M., Grillo, G., Sarnelli, P., Caterino, M. \& Greco, L., 1999: Ciliary motility at light microscopy: a screening technique for ciliary defects? Acta Paediatrica 88: 853-857.

SLEIGH, M.A., 1983: Kartagener's syndrome, ciliary defects and ciliary function. European Journal of Respiratory Disease 64: 157-161.

SPURR, A.R., 1969: A low-viscosity epoxy resin embedding medium for electron microscopy. Joumal of Ultrastructure Research 26: 31-43.

Sturgess, J.M. \& TurNer, J.A.P., 1990: The Immotile Cilia Syndrome. In V. Chernick (Ed.): Kendig's Disorders of the Respiratory Tract in Children. 5th edn. W.B. Saunders Company, Philadelphia: 675-768.

TAKASAKA, T., SATO, M. \& ONODERA, A., 1980: Atypical cilia of the nasal mucos. Annals of Otolaryngology 89: 37-45.

TOREMALM, N.G., 1983: What can we learn about ENT diseases from Kartagener's syndrome? European Journal of Respiratory Disease 64: 148-150.

Tsang, K.W.T., Tipoe, G., Sun, J., Ho, J.C.M., Lam, B., ZheING, L., OOI, G.C., IP, M. \& LAM, W., 2000: Severe bronchiectasis in patients with 'cystlike' structures within the ciliary shafts. American Joumal of Respiratory and Critical Care Medicine 161: 1300-1305.

WRIGHT, A., 1982: Giant cilia in the human organ of corti. Clinical Otolaryngology and Allied Sciences 7: 193-199.

Zahm, J.M., Pierrot, D., Hinnraskyk, J., Fuchey, C., Chevillard, M., Galllard, D. \& Puchelle, E., 1990: Functional activity of ciliated outgrowths from cultured human nasal and tracheal epithelia. Biorheology 27: 559--565.

(accepted 2 February 2004) 